\title{
Analysis of Fractional Systems using Haar Wavelet
}

\author{
Abdul Khader Valli T, Monica Mittal
}

\begin{abstract}
Wavelets are relatively new tool and have quite been thriving domain in mathematical research. Numerical solutions of differential and integral equations require development of accurate and fast algorithms based on wavelets. This is more pertinent for those problems having localized solutions, both in position and scale. Haar wavelet offers a promising solution bases due to simple mathematical expressions and multi-resolution properties. In this paper, A Haar wavelet based method to solve partial differential equations (PDE) modeling fractional systems is presented. Operational approach is based on representing various integro-differential mathematical operations in terms of matrices. In this article, firstly introduction of Haar wavelet and different operational matrices used for the analysis of fractional systems are presented. A modified computational technique is explained to solve variety of partial differential equations modeling systems of fractional order. This method achieves the solutions by solving Sylvester equation using MATLAB. Demonstrations are provided with the help of two illustrative examples by suitable comparisons with exact solutions.
\end{abstract}

Index Terms: Fractional partial differential equations (FPDE), Haar wavelet, Fractional calculus, Operational matrices, Sylvester equation

\section{INTRODUCTION}

Differential equations of fractional order are speculated from integer order derivatives, they are obtained by substituting derivatives of integer order by fractional ones. Compared with the derivatives of integer order, their benefits are ability to simulate dynamic systems and natural physical process more precisely [1]-[3]. For many researchers, fractional calculus has became the focus of interest. The recent investigation has unveiled its advantageous use in controlling and modeling many practical systems [4], [ 5]. For example, it is applied to continuum and statistical mechanics, fluid-dynamic traffic, economics, colored noise, signal processing, control theory [6]. The main difficulty of fractional differential equations is how to solve them, few techniques were suggested to solve differential equations of fractional order. Some of them are Generalized Differential Transform [7], [8] Variation Iteration technique [9], using Operational Matrix technique [10], Wavelet Method [11], [12], Finite Difference transform technique [13] and Adomian Decomposition Technique [14], [15], to name a few. But solutions are either difficult to be achieved or unachievable when the system is complex or of higher order. Therefore, there is a need for a computationally efficient and simple technique to achieve the solutions

This article focuses study on a variety of fractional partial differential equations (FPDE). Let partial differential equation of a system is described as

Revised Manuscript Received on June 15, 2019.

Abdul Khader Valli T, Department of Electrical Engineering, NIT Kurukshetra, Kurukshetra, India.

Monica Mittal, Department of Electrical Engineering, NIT Kurukshetra, Kurukshetra, India

$$
\frac{\partial^{x} y}{\partial x^{x}}+\frac{g^{9} y}{\partial t^{9}}=g\left(x_{x} t\right)
$$

And the subjected initial conditions are

$$
y(0, t)=y\left(x_{0}, 0\right)=0
$$

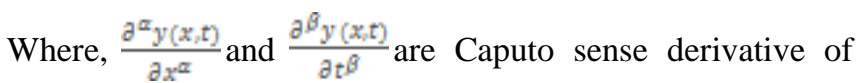
fractional order, $y(x, t)$ is unknown function, $\mathrm{g}(x, t)$ is the known continuous function. The following are the some of the methods for solving FPDE. Podlubny [16] applied Laplace Transform technique for getting numerical solution of FPDE with constant coefficients. Odibat [17] used generalized differential transform technique to obtain numerical solution of FPDE. In this paper, modified numerical method based on operational matrices of Haar wavelet is presented for solving FPDE.

Haar wavelet is a group of orthogonal functions with piecewise fixed values, have been studied in recent years and applied as a useful tool for analysis, synthesis and other control problems. Because of their simplicity in formulations, sparsity and multi resolution Haar wavelet has definite advantages for solving problems involving derivatives and integrals in an efficient manner [18].

In the next section required definitions, notations and review of the fractional differential calculus are explained

\section{DEFINITIONS}

\section{Definition 1}

Definition of fractional differential operator in the sense of Caputo is given as follows [16]

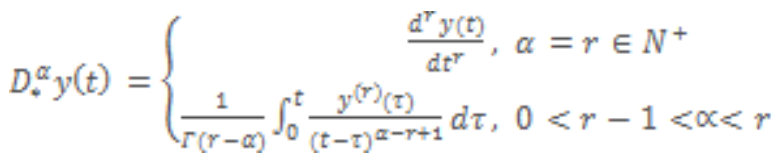

Where, $D_{*}^{\infty}$ is the differential operator of order $\alpha . y(t)$ be the function defined for $\mathrm{t}>0$ and $\mathrm{r}$ is the positive natural number. The derivative of fractional order $\alpha$ in Caputo sense can also

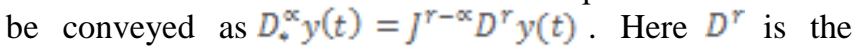
differential operator of order.

\section{Definition 2}

Fractional integral operator of Riemann-Liouville is defined as

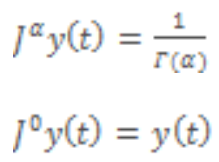

And its properties are

$f^{\infty} j^{\beta} y(t)=j^{\infty+\beta} y(t)$ 


$$
\begin{aligned}
& P^{\beta} \mathscr{Y}(t)=J^{\beta} J^{\mathbb{E}} \mathscr{Y}(t) \\
& l^{\mathbb{\alpha}_{t} \mathbb{Y}}=\frac{\Gamma(y+1)}{\Gamma(\alpha+\alpha+1)} t^{\mathbb{\alpha}+\mathscr{Y}}
\end{aligned}
$$

Where, $f^{\mathbb{E}}$ is the integral operator of order $\alpha . y(t)$ be the function defined for $\mathrm{t}>0$

\section{HAAR WAVELET OPERATIONAL MATRIX}

Haar functions are group of orthogonal functions. For $t \in[0,1]$ Haar functions are defined as, [21]

$$
h_{0}(t)=\frac{1}{\sqrt{x}}
$$

$h_{i}(t)=\frac{1}{\sqrt{m}}\left\{\begin{array}{cc}2^{\frac{j}{2}} & \frac{k-1}{2^{j}} \leq t \leq \frac{k-0.5}{2^{j}} \\ 2^{-\frac{j}{2}} & \frac{k-0.5}{2^{j}} \leq t<\frac{k}{2^{j}} \\ 0, & \text { otherwise }\end{array}\right.$

$$
i=0,1,2, \ldots m-1
$$

$m=2^{p+1} \quad$ And $p$ is the positive number. $i=2^{j}+k-1$ Where, $j$ and $k$ represents integer decompositions. Fig. 1 represents a set of Haar wavelet functions for $\mathrm{m}=4$.

Haar functions integration can be extended into Haar series with a matrix $\mathbb{P}_{\mathrm{n}}$ of size $\mathrm{m} \times \mathrm{m}$, it is known as integration operational matrix [17].

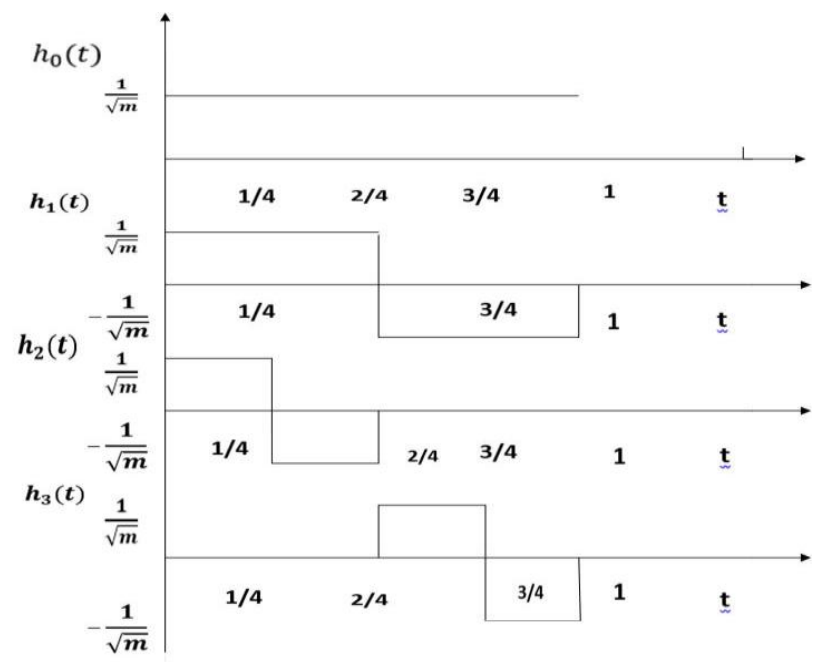

Fig. 1 Haar wavelets

The operational matrices of non-recursive formation are defined as [22]

$P_{m}=h_{m} * Q_{b m} * h_{m}^{-1}$

Where $Q_{b m}$ is the block pulse integral operational and is defined
$Q_{b m}=\frac{1}{m}\left[\begin{array}{cccc}1 / 2 & 1 & \ldots & 1 \\ 0 & \ldots x & \ldots x & \vdots \\ \vdots & 0 & 1 / 2 & 1 \\ 0 & \ldots & 0 & 1 / 2\end{array}\right]$

The block functions are defined in the intervalt $\in[0,1)$ as

$$
b_{i}(t)= \begin{cases}1_{v} & t \in\left[\frac{i}{m}, \frac{i+1}{m}\right]: i=0,1_{w n} m-1 \\ 0, & \text { otherwise }\end{cases}
$$

\section{SOLUTION OF THE FPDE}

This section illustrates modified methodology to get numerical solution for FPDE. Consider a function with two variables i.e. $Y\left(x_{x} t\right)=H^{\mathbb{T}}(x), C_{x} H(t)$,

Where, $\mathrm{C}$ is known as connection coefficient matrix. Integrating the function $Y\left(x_{o} t\right)$ w.r.t. variable $t$ Eq. (2) become

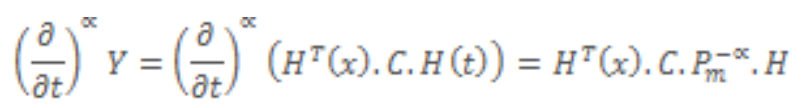

Similarly, integration w.r.t variable $x$ Eq. (2) become

$\left(\frac{\partial}{\partial x}\right)^{\beta} Y=\left(\frac{\partial}{\partial x}\right)^{\beta}\left(H^{T}(x) \cdot C \cdot H(t)\right)=H^{T}(x)_{*}\left(p_{m}^{-\beta}\right)^{T} C \cdot H(t)$

$\alpha$ and $\beta$ are fractional orders combining both equations it become

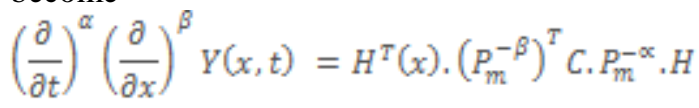

Eq. (1) is considered for illustration. Function $Y(x, t)$ is approximated with help of Haar functions as

$Y\left(x_{x} t\right)=H^{T}(x){ }_{n} c_{x} H(t)$.

Similarly the function $g\left(x_{w} t\right)$ is also expressed as

$g\left(x_{x} t\right)=H^{T}(x) \cdot G_{x} H(t)$

Where,

$G=\left[\begin{array}{cccc}g_{1,1} & g_{1,2} & \ldots & g_{1, n} \\ g_{2,1} & g_{2,2} & \ldots & g_{2, n} \\ \vdots & \ldots x & \ldots . & \vdots \\ g_{n_{1}, 1} & g_{m_{2},} & \ldots & g_{n_{n} n}\end{array}\right]$

Now by substituting Eq. (9), Eq. (10) and Eq. (11) into Eq. (1) we get

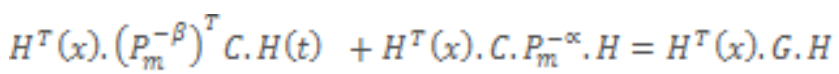

Simplifying the above equation converts into

$$
\left(P_{\mathrm{m}}^{-\beta}\right)^{T} C+C_{\mathrm{x}} P_{\mathrm{m}}^{-\mathrm{x}}=G
$$

Eq. (14) is a Sylvester equation which can be 
solved very easily by MATLAB.

\section{NUMERICAL EXAMPLES}

Example.1 [20]

Consider the non-homogeneous PDE

$\frac{\partial y}{\partial x}+\frac{\partial y}{\partial t}=1_{w} \quad x_{x} t \geq 0$

Initial conditions are given as $y\left(x_{0}, 0\right)=y(0, t)=0$

Exact solution of the given equation is given in [20]

$y\left(x_{w} t\right)= \begin{cases}t, & x \leq t \\ x_{w} & x>t\end{cases}$

The Fig. 2, Fig. 3 and Fig. 4 shows numerical solutions of $\mathrm{m}=8,16$ and 32 respectively. In Table 1 we are comparing numerical solutions of varying $\mathrm{m}$ with the exact solution. Also from Fig. 6 it is clear that the error in numerical solution is reduces by increasing the resolution of the Haar wavelet.

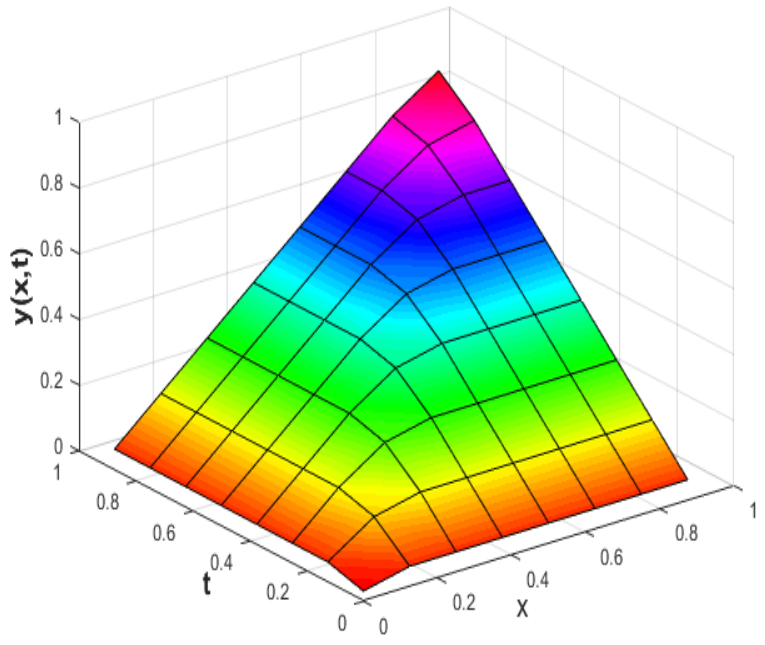

. Fig. 2 Numerical result of Example 1 with $m=8$

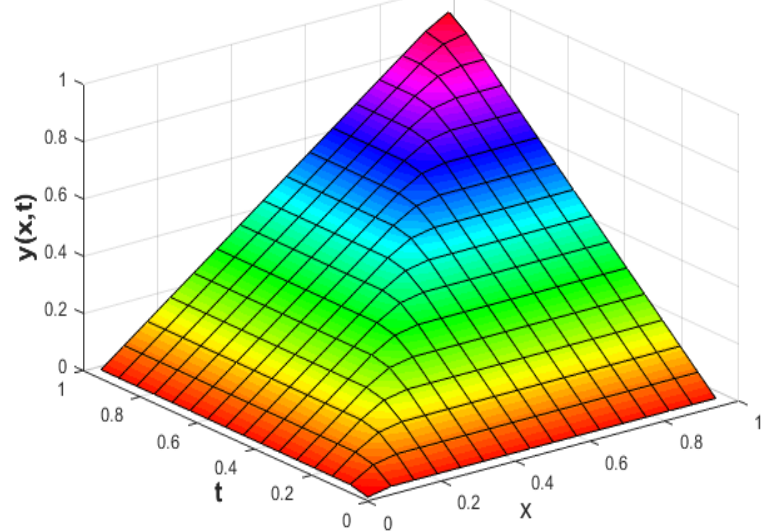

Fig. 3 Numerical result of Example 1 with $m=16$

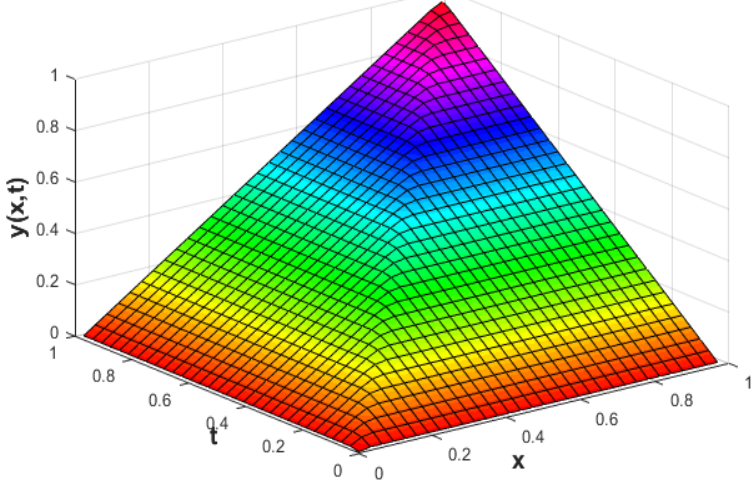

Fig. 4 Numerical result of Example 1 with $m=32$

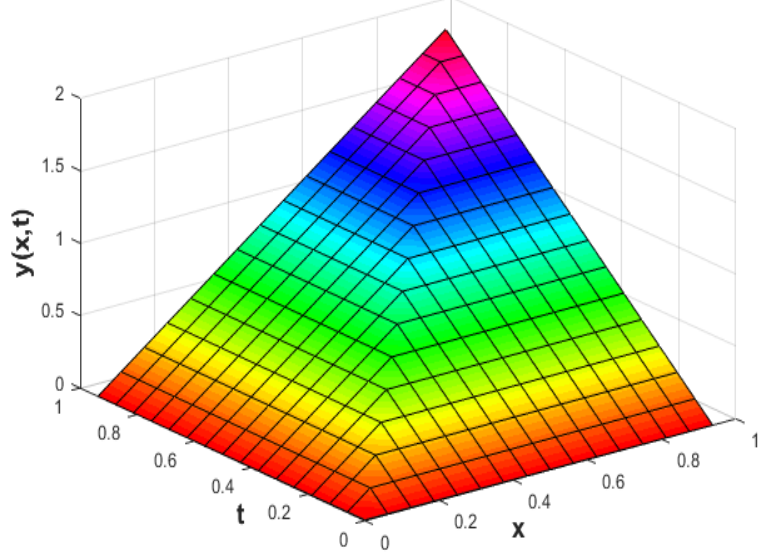

Fig. 5 Exact result of Example 1

Table 1. Comparison of numerical solution of $\mathrm{m}=8,16$ and 32 with Exact solution for Example 1

\begin{tabular}{lllll}
\hline$(\mathrm{x}, \mathrm{t})$ & $\mathrm{m}=32$ & $\mathrm{~m}=16$ & $\mathrm{~m}=8$ & $\begin{array}{l}\text { Exact } \\
\text { results }\end{array}$
\end{tabular}

\begin{tabular}{lllll}
$(0,0)$ & 0 & 0 & 0 & 0 \\
$(0.125,0.125)$ & 0.1328 & 0.1406 & 0.1562 & 0.125 \\
$(0.250,0.250)$ & 0.2578 & 0.2656 & 0.2813 & 0.250 \\
$(0.375,0.375)$ & 0.3828 & 0.3906 & 0.4062 & 0.375 \\
$(0.500,0.500)$ & 0.5078 & 0.5156 & 0.5313 & 0.500 \\
$(0.625,0.625)$ & 0.6328 & 0.6406 & 0.6563 & 0.625 \\
$(0.750,0.750)$ & 0.7578 & 0.7656 & 0.7813 & 0.750 \\
$(0.875,0.875)$ & 0.8828 & 0.8906 & 0.9063 & 0.875 \\
\hline
\end{tabular}

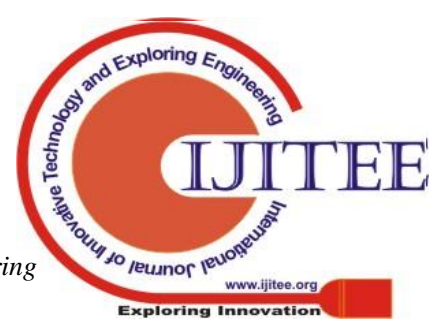




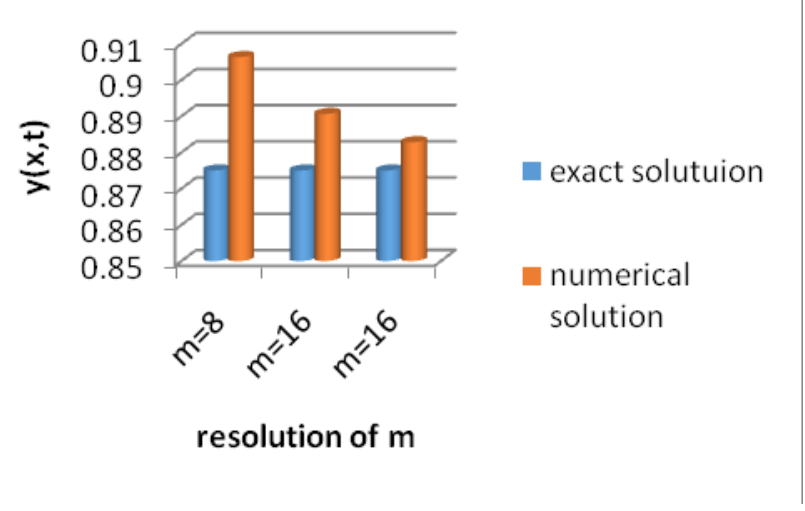

Fig. 6 Comparison of error of Haar and exact solutions at different $m$

Example.2 [20]

Given the following fractional FPDE

$\frac{\partial^{1 / 2} y}{\partial t^{1 / 2}}+\frac{\partial^{1 / 2} y}{\partial x^{1 / 2}}=1_{s} \quad x_{s} t \geq 0$

Initial conditions are given as $y(x, 0)=y(0, t)=0$ [20].By applying above method in MATLAB we get the numerical solutions of the problem are given in Fig. 6 and Fig. 7 respectively.

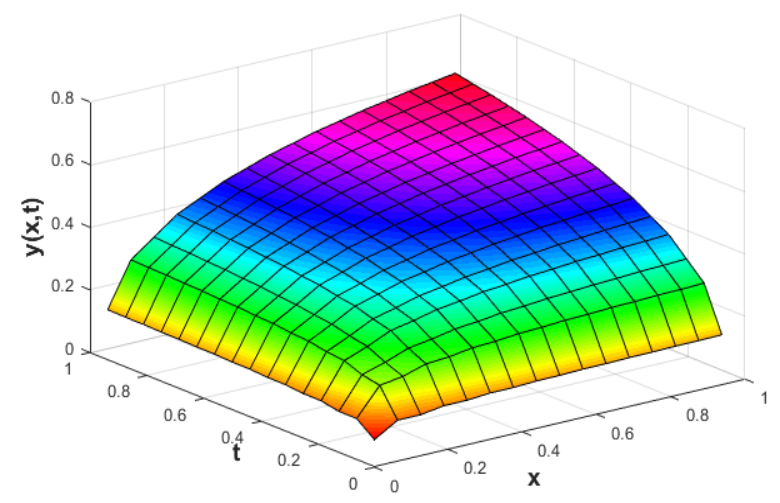

Fig. 6 Numerical result of $\operatorname{Exp} 2$ with $m=16$

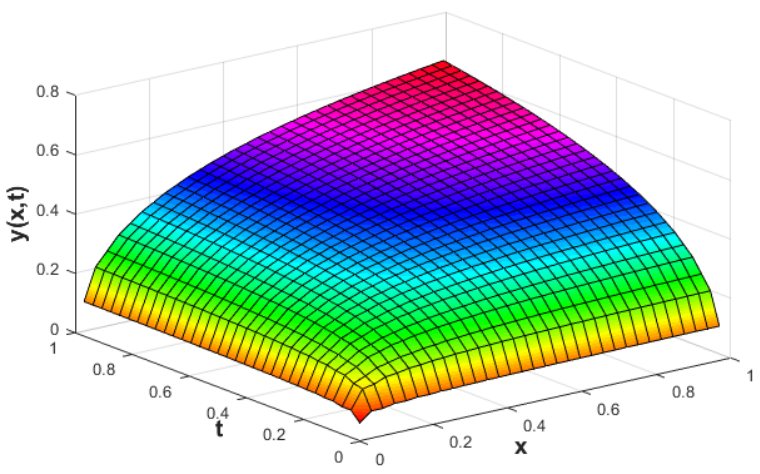

Fig. 7 Numerical result of Example 2 with $m=3$

This article uses the non recursive form of Haar integral operational matrix for solving partial differential equations of fractional order numerically. The highlight is that it converts problem into Sylvester equation. Advantages of this technique include (i) it is computer oriented and efficient; (ii) it is simple; (iii) the scope of application is wide. It is observed that by increasing $\mathrm{m}$, numerical solution approaches to exact solution. The numerically unstable problem never occurs in this technique.

\section{REFERENCES}

1. F. Liu, V. Anh, I. Turner, "Numerical solution of the space fractional Fokker-Planck equation," J. Comput. Appl. Math., 166 (2004), pp. 209-219.

2. S .A. EI-Wakil, A. Elhanbaly, M.A. Abdou, "Adomian decomposition method for solving fractional nonlineardifferential equations," Appl. Math. Comput., 182 (2006), pp. 313-324.

3. S. Chen, F. Liu, "Finite difference approximations for the fractional Fokker-Planck equation," Appl. Math. Model., 33 (2009), pp. 256-273.

4. A. Calderon, B. Vinagre, "Fractional order control strategies for power electronic buckconverters," Signal Process., 86 (2006), pp. 2803-2819.

5. M. Tavazoei, M. Haeri, "Chaos control via a simple fractional-order controller," Phys. Lett. A, 372 (2008), pp. 798-807.

6. Li Zhu, Qibin Fan, "Solving fractional nonlinear Fredholm integro-differentialequations by the second kind Chebyshev wavelet," Commun. Nonlinear Sci. Numer. Simul., 17 (2012), pp. 2333-2341.

7. Zaid M. Odibat, "A study on the convergence of variational iteration method," Math. Comput. Model., 51 (2010), pp. 1181-1192.

8. I.L. EI-Kalla, "Convergence of the Adomian method applied to a class ofnonlinear integral equations," Appl. Math. Comput., 21 (2008), pp. 372-376

9. M.M. Hosseini, "Adomian decomposition method for solution of nonlinear differential algebraic equations," Appl. Math. Comput., 181 (2006), pp. 1737-1744.

10.Shaher Momani, Zaid Odibat, "Generalized differential transform method for solving a space and time-fractional diffusion-wave equation," Phys. Lett. A, 370 (2007), pp. 379-387.

11.Zaid Odibat, Shaher Momani, "Generalized differential transform method: application todifferential equations of fractional order," Appl. Math. Comput., 197 (2008), pp. 467-477.

12.Abbas Saadatmandi, Mehdi Dehghan, "A new operational matrix for solving fractional-order differentialequations," Comput. Math. Appl., 59 (2010), pp. 1326-1336

13.Y. Zhang, "A finite difference method for fractional partial differential Equation," Appl. Math. Lett., 215 (2009), pp. 524-529

14.Y.M. Chen, Y.B. Wu, et al., "Wavelet method for a class of fractional convection-diffusionequation with variable coefficients," J. Comput. Sci., 1 (2010), pp. 146-149.

15.H. Jafari, S.A. Yousefi, “Application of Legendre wavelets for solving fractionaldifferential equations," Comput. Math. Appl., 62 (2011), pp. 1038-1045.

16.I. Podlubny, "Fractional Differential Equations," Academic press (1999).

17.Khushboo Kumari, Monika Mittal, "State analysis of singular time-varying bilinear systems using non-recursive Haar wavelet operational approach," 8th International Conference on Computing, Communication and Networking Technologies (ICCCNT-2017).

18.Zaid Odibat, Shaher Momani, "A generalized differential transform method for linear partial differential equations of fractional order," Appl. Math. Lett., 21 (2008), pp. 194-199.

19.Y.L. Li, N. Sun, "Numerical solution of fractional differential equations using thegeneralized block pulse operational matrix," Comput. Math Appl., 62 (2011), pp. 1046-1054.

20. Ming xu Yi , Jun Huang, Jin xia Wei, "Block pulse operational matrix method for solving fractional partial differential equation," Applied Mathematics and Computation, volume 221, 15 September 2013 Pages 121-131.

21.M Garg, L Dewan,

"Non-recursive Haar Connection Coefficients Based Approach for Linear

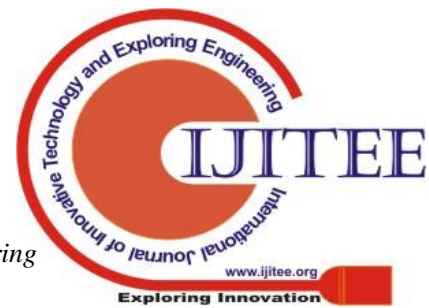


Optimal Control," Journal of Optimization Theory and Applications $153(2), 2012,320-337$.

22.M Garg, L Dewan, "A numerical method for linear ODEs using non-recursive Haar connection coefficients," International Journal of Computational science and Mathematics (IJCSM), 2010.

\section{AUTHORS PROFILE}

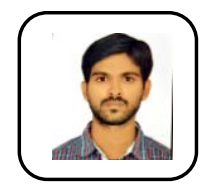

Abdul Khadervalli T, M.tech, is a research scholar at Department of Electrical engineering at the National Institute of technology Kurukshetra.

Dr. Monika Mittal has completed her Graduation in Electrical Engineering from M. M. M. Institute of Technology, Gorakhpur, India in 1992 and completed her Post Graduation and Doctorate from NIT KurukshetraShe has a teaching experience of about 25 years. She has authored about 50 research papers in International and National journals and conferences. She has interests in the areas of signal processing applications in control systems, computational algorithms, wavelets in control. Presently, she is working as Associate professor in Electrical Engineering Department, NIT Kurukshetra. 